Mots. Les langages du politique

Dire ou ne pas dire la « race » en France aujourd'hui

\title{
Réactions post-attentats en France et en Belgique : étude comparative des discours de l'exécutif politique
}

Political reactions after terrorist attacks in France and in Belgium: comparative discursive study of the executive

Reacciones post-atentados en Francia y en Bélgica: estudio comparativo de los discursos del ejecutivo político

\section{Albin Wagener}

\section{(2) OpenEdition}

\section{Journals}

Édition électronique

URL : https://journals.openedition.org/mots/23080

DOI : $10.4000 /$ mots. 23080

ISSN : 1960-6001

Éditeur

ENS Éditions

Édition imprimée

Date de publication : 20 mars 2018

Pagination : 111-130

ISSN : 0243-6450

Référence électronique

Albin Wagener, « Réactions post-attentats en France et en Belgique : étude comparative des discours de l'exécutif politique », Mots. Les langages du politique [En ligne], 116 | 2018, mis en ligne le 23 février 2020, consulté le 22 avril 2022. URL : http://journals.openedition.org/mots/23080 ; DOI : https:// doi.org/10.4000/mots.23080 


\section{Réactions post-attentats en France et en Belgique : étude comparative des discours de l'exécutif politique}

Les attentats du vendredi 13 novembre 2015 en France et du mardi 22 mars 2016 en Belgique ont déclenché une forte couverture médiatique en marquant durablement les esprits dans les deux pays. Dans les deux cas, le mode opératoire semble similaire à plus d'un titre : les deux capitales ont été touchées en plusieurs endroits le même jour, donnant lieu à des scènes de panique et à des réactions immédiates des deux États et de leurs classes politiques respectives. À la suite de ces attentats, la République belge et la République française sont intervenues pour mettre en œuvre des réactions politiques, d'ordre militaire et administratif, censées garantir la sécurité des citoyens et faire en sorte d'abaisser le risque d'attentat sur le territoire national. Dans ces deux séquences douloureuses, c'est d'abord le pouvoir exécutif qui se doit d'assurer cette double mission, notamment à travers deux hommes politiques : le président de la République François Hollande en France, et le Premier ministre Charles Michel en Belgique - en comptant également le roi des Belges, Philippe, relativement peu disert sur le sujet des attentats. Le présent article se donne pour objectif de comparer les deux réactions et la manière dont celles-ci traduisent une volonté politique spécifique et des représentations distinctes concernant la façon dont l'exécutif politique traite les attentats et propose d'y répondre par des réflexions et décisions de nature axiologique. À cette fin, il s'appuie sur un corpus de 12 discours officiels de ces hommes politiques : 6 pour François Hollande, 5 pour Charles Michel et 1 pour Philippe, roi des Belges. Tous ces discours ont été prononcés dans un intervalle d'un mois et demi au plus après l'attentat concerné. Il ne s'agit ici ni de prendre parti pour l'une ou l'autre option politique, ni d'évacuer la question des spécificités politiques et constitutionnelles des deux pays : nous sommes conscient du fait qu'un président et un Premier ministre sont investis d'auras et de missions fort différentes et que les systèmes politiques sont distincts en France (Lazorthes, 2006) et en Belgique (Uyttendaele, 2011), mais ils représentent ici tous deux de manière évidente le pouvoir exécutif de leurs pays respectifs. L'analyse de corpus que nous 
proposons se fonde sur les résultats extraits des logiciels Wordsmith et Iramuteq, et se trouve complétée par une analyse de discours pragmatique appliquée à des corpus de type institutionnel ou politique.

\section{L’analyse de discours comme accès aux représentations}

\section{Une théorie systémique du discours}

Notre travail s'inscrit dans une théorie systémique du discours, qui permet d'allier la complexité inhérente à tout phénomène communicationnel à la nécessaire circulation des représentations de sens situés dans un interdiscours riche de références et de constructions sociétales (Garric, Longhi, 2013). Une telle théorie du discours prend appui sur la philosophie pragmatique du discours et notamment la diffusion, la transmission et la réception d'un sens commun propre à une communauté de sens donnée, particulièrement dans le contexte institutionnel et politique :

À l'échelle pratique réelle, un sujet-acteur participe de la formation sociale non pas par le biais de l'ensemble des institutions de sens qui la constituent, mais uniquement à partir de certaines des communautés de sens qui matérialisent chaque institution de sens. Du point de vue du sujet-acteur, l'ensemble restreint des communautés de sens dans lesquelles il est concrètement impliqué détermine son écosphère. (Sarfati, 2014, p. 23)

Dans ce cas précis, la communauté de sens à laquelle s’adressent les représentants de l'exécutif de France et de Belgique est la communauté nationale, dans la mesure où celle-ci se retrouve obligée de faire corps face à un événement qui vient de la toucher de manière inattendue et violente. L'institution politique exécutive a alors pour but de commenter solennellement le drame en participant à la création d'un moment discursif, de tenter d'apporter des éléments d'explication ou de réponse, et de rassurer en proposant des dispositifs qui ont pour objectif intentionnel d'empêcher les actes terroristes ou d'en limiter la portée :

En effet, dans l'institution, les locuteurs, sommés de répondre à des exigences complexes voire contradictoires, obéissent à une injonction plus générale : organiser la polyphonie, réduire la dissonance, produire la cohérence. (Krieg-Planque, Oger, 2010, p.93)

Dans le cadre de discours politiques institutionnels, cette injonction à produire de la cohérence est importante : elle vise à faire corps, voire à faire «nation », afin de répondre à une menace violente. Cette contrainte est d'autant plus forte du fait de l'aspect foncièrement public des croyances et institutions sociales (Boudon, 1995, p.560-561), particulièrement dans un moment où l'événement discursif fait écho à un acte émotionnel fort et partagé, ne serait- 
ce que par l'amplification de la caisse de résonance médiatique. Cependant, comme l'homme d'État se trouve également être un acteur politique, les réactions discursives sont nécessairement enracinées dans un déroulé idéologique qui propose ou impose des choix à ceux qui lisent ou entendent ces réactions :

Indeed, the process of mediating social reality involves making choices as to what aspects of this reality will be proximized and to what extent. And most of these choices are discursive in nature. The motivations behind them and the implications they might have for the public perception of social reality matter. The former are inextricably linked with notions of power and ideology, while the latter impact on identity construction, negotiation and contestation, along with the (...) mediated quasi-status functions and collective agreement ${ }^{1}$. (Kopytowska, 2015, p.144)

De ce fait, la réponse de l'État à un événement disruptif est une réponse politique portée par des choix qui s'annoncent dans les discours, proposant ainsi des représentations du fait social, et cherchant alors à provoquer des effets sur la classe politique, les médias et les citoyens. En tant que vecteur, co-créateur et réceptacle de représentations, le discours institutionnel ou politique charrie un ensemble complexe d'items qu'il convient d'analyser à l'aide d'un outillage méthodologique pragmatique et d'une entrée par les déictiques.

\section{Méthodologie d'analyse systémique du discours}

La méthodologie que nous proposons a un triple ancrage, dans la lexicométrie des corpus (Baker, 2006), dans la sémantique des possibles argumentatifs (Galatanu, 2009) et dans la proximisation pragmatique (Cap, 2013). Cette triple assise offre la possibilité d'engager une analyse de discours susceptible de mettre en relief trois aspects essentiels liés aux productions et contextes discursifs :

- la fréquence des occurrences lexicales et de leurs collocations, afin d'obtenir des résultats statistiques suffisamment significatifs, grâce à l'utilisation de logiciels tels qu'Iramuteq et Wordsmith, et afin d'extraire des données à partir desquelles pourra se faire l'analyse sémantique et pragmatique;

- une approche du sens dans les représentations, reliée notamment aux formes lexicales, à la fois en tant qu'entités isolées dans le discours, mais également en interaction constante avec un environnement sémantique qui produit des effets de circulation et de compréhension pragmatiques;

1. «En effet, le processus de médiatisation de la réalité sociale implique le fait de faire des choix à propos des aspects de la réalité qui seront proximisés, et de quelle manière ils le seront. La plupart de ces choix sont, par nature, discursifs. Les motivations qui s'y logent comptent, autant que les implications que ceux-ci peuvent avoir pour la perception publique de la réalité sociale. Les premières sont inextricablement liées aux notions de pouvoir et d'idéologie, tandis que les secondes ont un impact sur la construction, la négociation et la contestation de l'identité, en même temps que les fonctions médiatisées quasi statutaires et l'assentiment collectif » (les traductions des citations sont de nous). 
- une compréhension du positionnement du discours, notamment dans le rapport entre énonciateur et récepteur, plus particulièrement dans la mise en scène, au sein même du discours, de la réception émotionnelle attendue de ses complexités déictiques, axiologiques et modales.

Une telle approche permet de délimiter la topographie des espaces représentationnels contenus dans le corpus et les sous-corpus :

Les espaces représentationnels donnent une clef de lecture originale aux prises de parole qui les sous-tendent, car ils permettent de «voir» le monde que le locuteur construit et les relations qu'il entretient avec les éléments qui le composent. Chemin faisant, ils offrent la possibilité d’identifier une partie des connaissances convoquées lors de l'interprétation. [...] La figure obtenue reconstruit un prototype des productions prises en compte, elle en subsume les éléments les plus représentatifs, indépendamment des variations thématiques. (Bendinelli, 2011, p.110)

La figuration des espaces représentationnels est importante et sa construction peut être modélisée en suivant trois étapes :

- dans un premier temps, la présentation des occurrences les plus fréquentes dans le corpus, ainsi que leurs singularités par variations de sous-corpus;

- dans un second temps, l'analyse des occurrences et de leurs collocations lexicales les plus fréquentes, afin de comprendre le poids des nœuds sémantiques qui jalonnent le corpus et ses sous-corpus;

- dans un troisième temps, enfin, l'étude pragmatique du texte, notamment à travers l'analyse des espaces déictiques et des particularités modales et axiologiques.

Dans le cas précis de cette étude, nous estimons que cette méthodologie doit permettre de mettre en lumière les spécificités et différences des discours post-attentats produits par les exécutifs français et belge, et d'y apporter des propositions d'interprétation.

\section{Étude du corpus : occurrences, fréquences et collocations}

\section{Présentation et analyse lexicométrique}

Comme nous l'avons précisé au début de notre étude, le corpus que nous étudions réunit 12 textes distincts, répartis de manière égale entre les exécutifs nationaux :

- 6 pour la France, produits exclusivement par le président de la République François Hollande, dont 3 à dimension internationale ;

- 6 pour la Belgique, produits majoritairement par le Premier ministre Charles Michel ( 5 textes), mais également par le roi Philippe (1 seul texte), tous à dimension nationale. 
Outre ces particularités, les textes présents au sein des deux sous-corpus se répartissent comme suit :

- France, 14/11/2015 : déclaration de François Hollande pour le Conseil de défense;

- France, 16/11/2015: discours au Congrès de François Hollande;

- France, 24/11/2015: discours de conférence de presse à la Maison-Blanche auprès de Barack Obama par François Hollande;

- France, 25/11/2015 : déclaration conjointe avec Angela Merkel par François Hollande;

- France, 26/11/2015 : discours à Moscou auprès de Vladimir Poutine par François Hollande;

- France, 27/11/2015 : hommage national de François Hollande aux victimes des attentats à l'hôtel des Invalides;

- Belgique, 22/03/2016: communiqué de presse de Charles Michel au nom du gouvernement fédéral;

- Belgique, 22/03/2016 : discours à la conférence de presse gouvernementale par Charles Michel;

- Belgique, 22/03/2016: discours du roi Philippe;

- Belgique, 24/03/2016: discours d'hommage aux victimes des attentats par Charles Michel;

- Belgique, 20/04/2016 : déclaration commune du gouvernement et des représentants des cultes et de la laïcité par Charles Michel;

- Belgique, 01/05/2016: discours de la réouverture de l'aéroport de Zaventem par Charles Michel.

Les premiers éléments statistiques révélés par Iramuteq font état d'un corpus réunissant 11841 occurrences et 1767 formes différentes. La première différence entre les deux corpus français et belge est très importante, puisqu'elle présente un net déséquilibre entre le volume des déclarations de l'exécutif : alors que le sous-corpus de l'exécutif français totalise 10063 occurrences et 1557 formes différentes, le sous-corpus de l'exécutif belge ne totalise que 1778 occurrences et 573 formes distinctes. Cette première singularité dénote une différence concernant les réactions post-attentats : là où les discours de l'exécutif français sont longs et nourris de détails, les discours de l'exécutif belge sont nettement plus sobres et ne contiennent pas le même type de déclarations, comme nous pourrons le voir ultérieurement.

Les termes les plus mobilisés dans les sous-corpus liés respectivement aux réactions françaises et belges présentent également un nombre important de différences. Au sein des discours et des représentations convoquées, notamment par François Hollande et Charles Michel, de réelles divergences se font jour concernant les réactions politiques de l'exécutif, et mettent en lumière deux manières fort singulières de traiter le moment post-attentat. Nous choisissons ici, pour les deux sous-corpus, de sélectionner les 20 termes les plus 
récurrents grâce à Iramuteq, en sélectionnant notamment des lexèmes sémantiquement signifiants - à ce titre, nous faisons le choix d'évacuer directement les prépositions ou autres termes tels que «et» ou «de», qui sont systématiquement surreprésentés dans les corpus en langue française, mais qui ne fournissent que peu d'indices représentationnels.

\begin{tabular}{l|c|c}
\hline France & 73 & $\mathrm{nr}$ \\
\hline pays & 43 & nom \\
\hline terroriste & 36 & nom \\
\hline terrorisme & 31 & nom \\
\hline Daech & 29 & $\mathrm{nr}$ \\
\hline monde & 25 & nom \\
\hline Syrie & 23 & nr \\
\hline français & 22 & adj \\
\hline agir & 21 & ver \\
\hline vivre & 20 & ver \\
\hline venir & 20 & ver \\
\hline guerre & 20 & nom \\
\hline État & 19 & nom \\
\hline unir & 19 & ver \\
\hline acte & 19 & nom \\
\hline président & 18 & nom \\
\hline pari & 18 & nom \\
\hline prendre & 16 & ver \\
\hline fois & nom \\
\hline frapper & ver \\
\hline & 17 & \\
\hline
\end{tabular}

Tableau 1 : 20 premières occurrences du sous-corpus France

\begin{tabular}{|c|c|c|}
\hline victime & 9 & nom \\
\hline liberté & 9 & nom \\
\hline famille & 9 & nom \\
\hline aéroport & 9 & nom \\
\hline sécurité & 8 & nom \\
\hline pays & 7 & nom \\
\hline proche & 6 & adj \\
\hline moment & 6 & nom \\
\hline face & 6 & nom \\
\hline attentat & 6 & nom \\
\hline vie & 5 & nom \\
\hline société & 5 & nom \\
\hline service & 5 & nom \\
\hline penser & 5 & ver \\
\hline nombreux & 5 & adj \\
\hline exprimer & 5 & ver \\
\hline ensemble & 5 & adv \\
\hline démocratie & 5 & nom \\
\hline également & 4 & $\mathrm{adv}$ \\
\hline Zaventem & 4 & $\mathrm{nr}$ \\
\hline
\end{tabular}

Tableau $2: 20$ premières occurrences du sous-corpus Belgique

Malgré l'écart évident entre le nombre total d'occurrences, comme nous l'avons déjà souligné, la différence de ton et de sens commun est très importante. Nous pouvons d'ores et déjà isoler en effet les spécificités axiologiques suivantes, pour ce qui est des réactions des exécutifs français et belge aux attentats qui ont frappé leurs capitales respectives :

- Les discours de l'exécutif français font immédiatement référence à l'entité nationale et aux institutions ("France», 73 occurrences; "président», 18 occurrences ; «français », 22 occurrences ; «pays », 43 occurrences ; "État», 19 occurrences), à la guerre face au terrorisme («terroriste», 36 occurrences; «terrorisme», 31 occurrences; «Daech », 29 occurrences; « guerre », 20 occur- 
rences; "frapper», 16 occurrences; «Syrie», 23 occurrences) et à la mobilisation nationale («agir», 21 occurrences; «vivre», 20 occurrences; « unir», 19 occurrences).

- Les discours de l'exécutif belge, en revanche, se tiennent très loin d'une telle rhétorique patriote ou guerrière et se positionnent nettement autour du vivre-ensemble ("liberté», 9 occurrences; "vie», 5 occurrences; "société », 5 occurrences; «ensemble», 5 occurrences; "démocratie», 5 occurrences) ou du dramatique moment de l'attentat lui-même ("victime», 9 occurrences; «famille», 9 occurrences; "aéroport», 9 occurrences; "proche», 6 occurrences; «moment», 6 occurrences; «attentat», 6 occurrences).

Dans le cas de la Belgique, le nom même du pays est totalement absent des 20 premières occurrences, et toute référence à l'espace national semble absente. Ces premiers indices lexicographiques ne sont pas dénués de sens ni d'intérêt, et permettent de donner une rapide esquisse des représentations politiques véhiculées par les responsables de l'exécutif en France et en Belgique à la suite des dramatiques événements qui secouent leurs nations respectives.

La présence de discours à portée internationale dans le sous-corpus français n'explique pas cette différence de traitement : si nous ne nous centrons que sur les trois discours à portée nationale, nous parvenons en effet à des résultats lexicométriques analogues, à savoir : toujours une focalisation sur l'entité nationale ("France », 52 occurrences ; "pays », 30 occurrences; «français», 19 occurrences; "État», 16 occurrences), la guerre face au terrorisme ("terroriste», 23 occurrences; "terrorisme», 18 occurrences; "Daech», 11 occurrences; « guerre», 20 occurrences ; «armée», 12 occurrences ; «Syrie», 10 occurrences) et la mobilisation nationale («vivre», 16 occurrences; «agir», 8 occurrences; « unité », 8 occurrences).

Cette précision est utile : elle nous permet de signaler une relative cohérence lexicologique et, de ce fait, représentationnelle entre les discours de François Hollande, que ceux-ci aient une portée internationale ou simplement nationale. Cette relative stabilité interdiscursive du président français va, comme nous allons le constater, le poser en tant que «chef de guerre », tant aux niveaux national qu'international.

\section{Cooccurrences lexicales et nœuds sémantiques}

Pour mieux comprendre la manière dont les textes tissent les réseaux discursifs représentationnels, nous proposons de croiser le simple comptage des 10 premières occurrences de lexèmes ou lemmes pour chaque sous-corpus avec une mise en situation de ceux-ci au sein de leurs environnements de collocations lexicales. Afin de respecter la différence de taille entre les deux souscorpus, les cooccurrences lexicales conservées pour la France n’iront pas en 
dessous de 3 cooccurrences alors que la limite de cooccurrences lexicales pour la Belgique ne pourra pas aller en deçà de 2 cooccurrences. Pour ce faire, nous choisissons d'utiliser le logiciel de lexicométrie Wordsmith et de présenter une vue d'ensemble en croisant ce que nous appelons les occurrences A (soit les occurrences immédiatement décomptées comme plus fréquentes dans le corpus) et les occurrences $B$ (soit les occurrences visibles grâce à l'opération de calcul des formes de collocation). Cette méthodologie permet, à travers la lecture des occurrences B, qui n'apparaissent qu'indirectement en tant que points des réseaux de sens représentationnel, d’isoler les principaux nœuds sémantiques des sous-corpus.

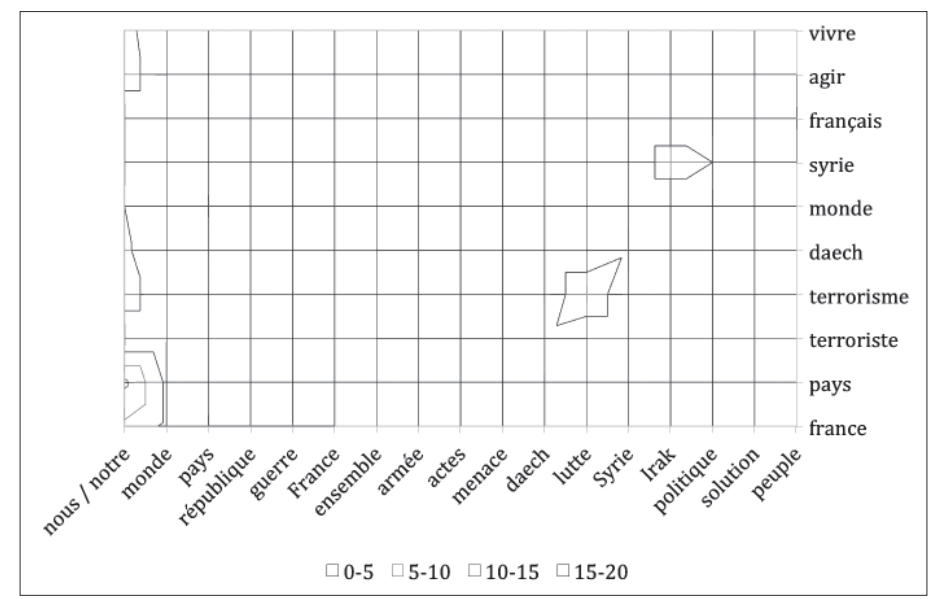

Schéma 1 : topographie des cooccurrences du sous-corpus France

Pour ce qui est des réactions de l'exécutif français, représenté ici exclusivement par les prises de parole du président François Hollande, la topographie du sous-corpus présente des particularités intéressantes, et regroupe les nœuds sémantiques suivants :

- «notre/nous» et «pays»: 16 cooccurrences;

- «lutte» et «terrorisme»: 10 cooccurrences;

- «notre/nous» et «France»: 9 cooccurrences;

- «notre/nous» et «terrorisme»: 8 cooccurrences;

- «notre/nous» et «agir»: 8 cooccurrences;

- «Syrie» et «Irak»: 8 cooccurrences.

Ces six nœuds sémantiques permettent d'obtenir une image représentationnelle du sous-corpus fourni par les déclarations du président François Hollande, notamment à travers une forte implication de l'espace déictique interne «nous/notre», que nous étudierons plus précisément par la suite. Dans tous les cas de figure, ce «nous» rassembleur est à la fois inclusif (avec «France» 
et "pays») et exclusif (avec «terrorisme), avec un verbe à forte valeur axiologique et modale, à savoir "agir», qui se retrouve au centre de ces deux pôles d'inclusion et d'exclusion.

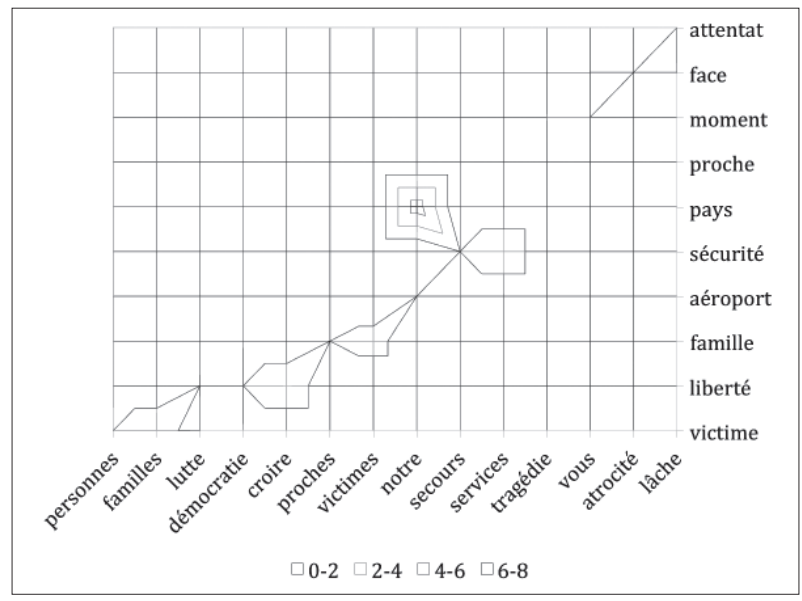

Schéma 2 : topographie des cooccurrences du sous-corpus Belgique

Le sous-corpus produit par l'exécutif belge propose une réaction représentationnelle tout à fait différente face à l'attentat. Les nœuds sémantiques mis en valeur par le Premier ministre Charles Michel et le roi Philippe sont les suivants :

- «notre» et «pays»: 7 cooccurrences;

- «victimes» et «familles» : 7 cooccurrences (en additionnant les mentions indirectes de ces lexèmes dans leur rôle d'occurrence $B$ );

- «croire» et «liberté »: 4 cooccurrences;

- «services» et «sécurité»: 4 cooccurrences.

Comme nous pouvons le constater, les représentations mises en circulation par l'exécutif belge sont bien différentes de celles de l'exécutif français; s’il est fait mention du pays et de l'espace déictique interne (sans toutefois nommer la Belgique), l'accent est fortement mis sur les victimes de l'attentat, suivi ensuite par l'espoir de la liberté comme réponse à l'attentat, et les remerciements pour le travail des services de sécurité pendant les événements tragiques. Cette analyse statistique des cooccurrences et de leurs produits, à savoir les nœuds sémantiques, permet de proposer, par des tissus sémantiques issus de la concaténation des nœuds, les schémas suivants, qui permettent de réduire les principaux pôles représentationnels des deux sous-corpus. 


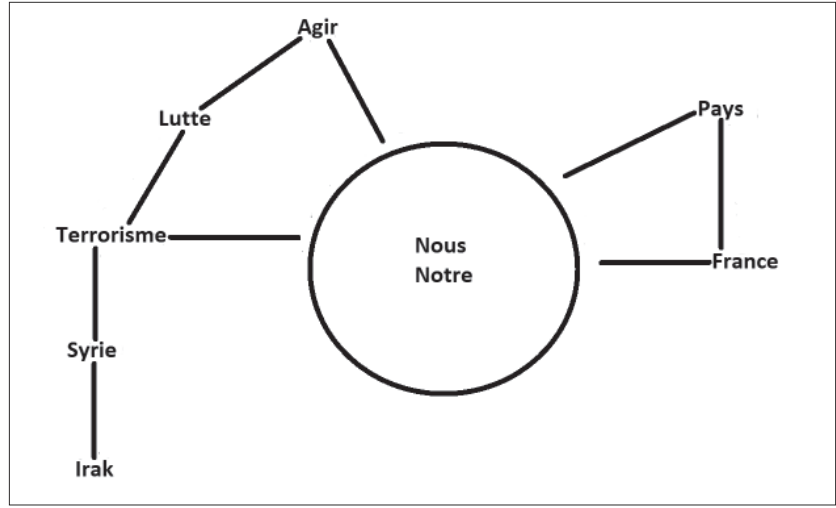

Schéma 3 : tissu sémantique et nœuds sémantiques du sous-corpus France

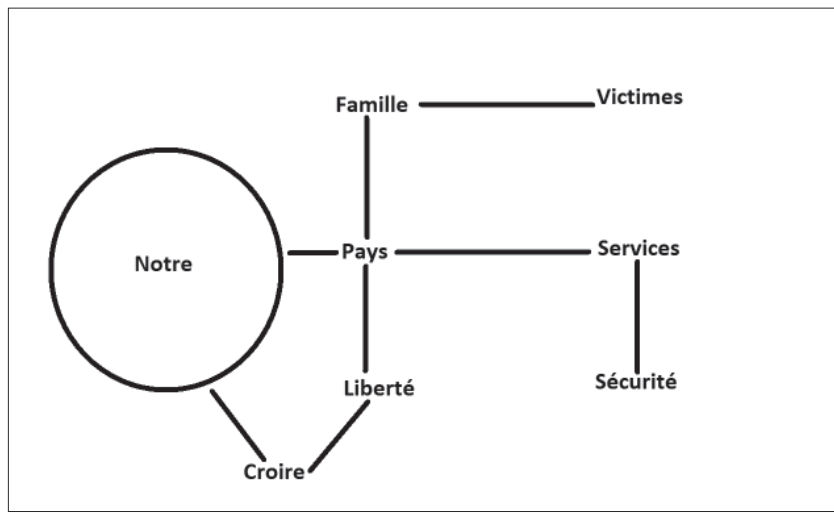

Schéma 4 : tissu sémantique et nœuds sémantiques du sous-corpus Belgique

\section{Interprétation sémantico-pragmatique}

\section{Présentation des classes sémantiques}

La partie lexicographique de notre étude permet d'envisager la statistique lexicale comme point de départ : toutefois, elle ne saurait constituer la seule façon d'étudier un corpus aussi complexe, composé de sous-corpus divers et singuliers, aussi bien à travers les axes représentationnels des locuteurs que par l'histoire politique et institutionnelle des deux nations qu'ils représentent. Mais la lexicométrie ne saurait suffire : point d'entrée de l'analyse de discours, elle ne permet pas de livrer suffisamment d'interprétations sur le sens en circulation et la manière dont ce sens s'organise autour d'effets pragmatiques. 
Comme les langages politiques et institutionnels sont particulièrement riches de ce point de vue, il convient alors d'organiser les sous-corpus présentés à travers les classes de correspondances lexicales autour desquelles ils se structurent, notamment grâce à l'utilisation du logiciel Iramuteq. Ces classes à portée pragmatique sont organisées en dendrogrammes, comme pour le souscorpus produit par François Hollande, dont nous proposons l'analyse suivante.

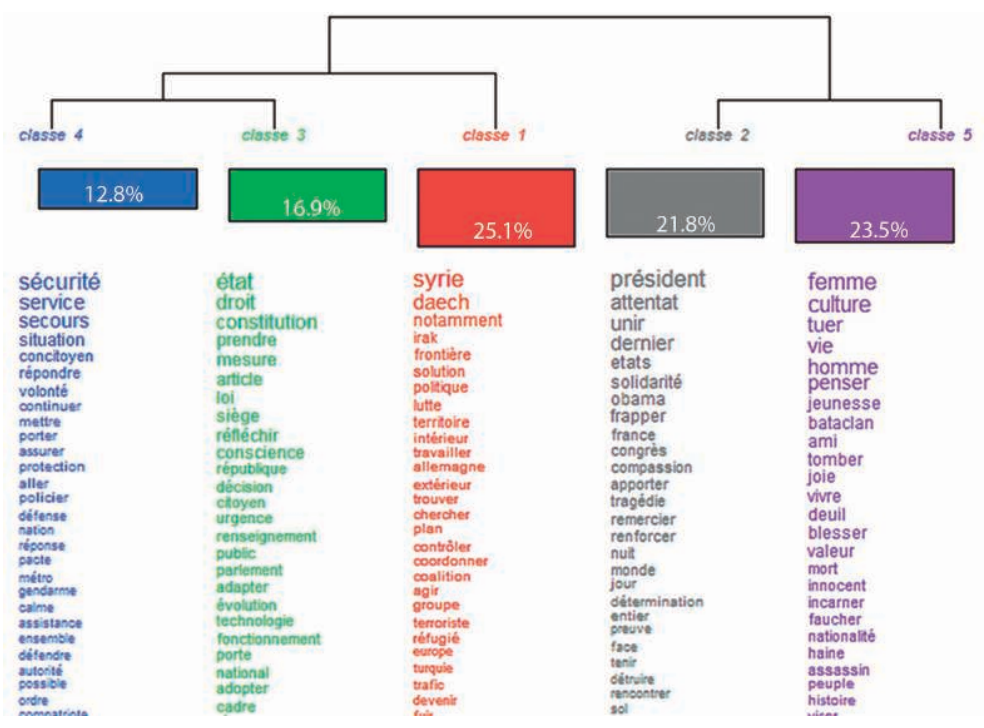

Schéma 5 : dendrogramme des classes de correspondances lexicales du sous-corpus France

Le logiciel Iramuteq propose le découpage du sous-corpus en deux grands embranchements, qui se subdivisent ensuite en cinq classes distinctes, que nous présentons comme suit :

- Classe 4: cette classe est celle de la lutte internationale contre le terrorisme, dans la mesure où elle réunit plusieurs acteurs (Allemagne et Turquie en tête) en nommant des territoires propices à l'évolution du terrorisme islamique (Irak, Syrie, Liban), mais également Daech comme acteur terroriste majeur;

- Classe 3 : cette classe, directement liée à la classe 4, est celle de la lutte intérieure contre le terrorisme, en positionnant l'État comme entité délimitée, chargée de devoir effectuer des contrôles à ses frontières, notamment dans le cadre européen (puisque c'est bien dans ce cadre que se situe la France);

- Classe 1 : ici il s'agit, en lien avec les deux classes précédentes, de positionner l'État en tant qu'entité institutionnelle et politique, dotée d'une légitimité et susceptible de se donner les moyens légaux pour pouvoir se positionner dans la lutte contre le terrorisme et protéger sa population;

- Classe 5 : cette classe fort différente est centrée sur des valeurs ciblées 
par les terroristes lors des attentats de Paris, mais également sur des victimes elles-mêmes et la culture que celles-ci faisaient vivre au moment du drame;

- Classe 2 : voisine de la classe 5 , cette dernière classe met en lumière le positionnement de la France dans le monde et le message qu'elle souhaite délivrer par-delà ses frontières de manière solennelle face à ce qu'elle a vécu.

Comme nous pouvons le constater, les trois premières classes, regroupées au sein du même embranchement, représentent à elles seules environ $60 \%$ des productions, ce qui permet de confirmer le premier tissu de sens qui positionne la France comme un État engagé dans la lutte contre le terrorisme. Le second embranchement, plus directement lié aux valeurs et à la culture du pays, apparaît comme moins emblématique ; selon nous, cela est précisément lié au rôle du chef d’État endossé par le président Hollande, notamment en matière de lutte contre le terrorisme depuis le début de son quinquennat, plus spécialement au-delà des frontières françaises. Ainsi, le découpage par classes livré par Iramuteq permet de faire le portrait d'un État français qui se retrouve dans une position à visée internationale lorsqu'il est victime d'un attentat, et qui l'assume fermement - ce qui est évidemment soutenu par le fait que trois des discours du corpus sont à portée internationale.

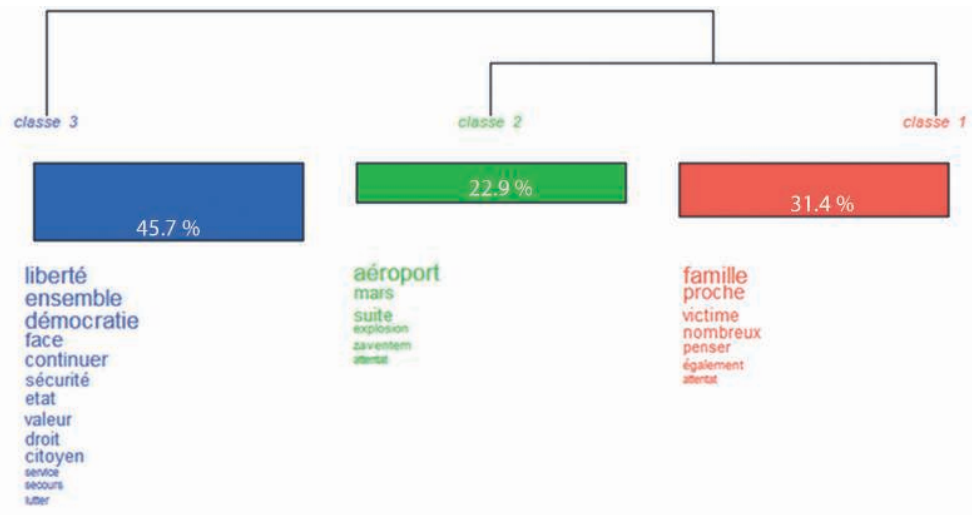

Schéma 6 : dendrogramme des classes de correspondances lexicales du sous-corpus Belgique

Si le sous-corpus plus restreint lié à l'exécutif belge montre qu'Iramuteq ne peut pas isoler des classes aussi riches que celles du sous-corpus lié à l'exécutif français, il n'en reste pas moins que trois classes peuvent être directement mises en relief lorsque l'on étudie les discours du Premier ministre Charles Michel et du roi Philippe de Belgique :

- Classe 3: dès l'étude de cette classe, le contraste entre les représentations liées à la réaction de l'exécutif belge et celui de la France est frappant. En effet, 
la première et la plus importante classe de ce sous-corpus est d'abord centrée sur l'idéal démocratique, et notamment le vivre-ensemble qui doit continuer de manière indéfectible après les attentats de Bruxelles;

- Classe 2 : cette classe est la plus descriptive du sous-corpus et présente avant tout les faits eux-mêmes, notamment ceux qui sont liés à l'explosion survenue à l'aéroport de Zaventem;

- Classe 1 : directement reliée à la classe 2 d'après Iramuteq, cette dernière classe a d'abord pour objet l'attention portée aux victimes, à leurs familles et à leurs proches.

Ainsi, alors que la réponse proposée par l'exécutif français est celle de l'action internationale dans la lutte contre le terrorisme et du contrôle aux frontières, que les textes soient de portée nationale ou internationale, les discours nettement plus courts de l'exécutif belge portent avant tout une autre forme de réponse, moins guerrière : celle d'une poursuite du mode de vie démocratique comme seule riposte possible face au terrorisme. Elle constitue de fait une différence intéressante entre les deux sous-corpus : là où le socialiste François Hollande n'utilise pas le mode de vie et les valeurs du vivre-ensemble comme réponse au terrorisme, le Premier ministre Charles Michel, homme politique libéral de centre droit, qui évolue dans un environnement gouvernemental de coalitions politiques, met l'accent sur ces fondamentaux de la vie démocratique sociétale. Cette différence souligne également un autre écart : la France représente une puissance politique et militaire engagée de manière internationale dans un certain nombre de foyers islamistes, là où la Belgique ne peut pas se fonder sur un statut de puissance mondiale ou fortement militarisée pour répondre à la menace terroriste. De ce point de vue, le sous-corpus de l'exécutif belge relève peut-être d'une forme de réalisme politique : il n'est pas interdit de penser que la Belgique ne dispose pas réellement d'autres réponses que celle de son mode de vie pour répondre à la menace terroriste, même s’il peut paraître étonnant de constater que ni Charles Michel ni le roi Philippe ne mentionnent le fait que la Belgique est touchée par l'implantation d'individus liés au terrorisme en Europe sur son sol (O’Brien, 2016). De ce point de vue, ce silence peut poser question - ce qui ne signifie pas, d'ailleurs, que la France ne soit pas dans le même cas de figure, malgré un déploiement de troupes international pour s'engager dans la lutte contre le terrorisme.

\section{Modélisation des espaces déictiques}

Afin de terminer notre étude de corpus, nous proposons de dépeindre les relations au sein de l'espace représentationnel sémantique, afin de pouvoir isoler les mécanismes pragmatiques à l'œuvre dans les deux sous-corpus. Pour ce faire, nous mettons en rapport les espaces déictiques internes et externes aux sous-corpus, à travers des curseurs axiologiques et modalisateurs, ce qui 
permet d'affiner l'analyse au-delà des classifications sémantiques proposées précédemment. Ce modèle de spatialisation cognitive des représentations est notamment conçu et mis en œuvre par les travaux de Paul Chilton, sur la base d'axes temporel, spatial et modal, puis, plus tard, axiologique :

Entities and the relations among them may be represented as physically necessary, socially imposed or as morally imperative. There is a strong spatial element in this dimension also. [...] We are suggesting that in processing any discourse people "position" other entities in their "world" by "positioning" these entities in relation to themselves along (at least) three axes, space, time and modality. The deictic centre (the Self, that is, I or we) is the "origin" of the three dimensions. Other entities (arguments of predicates) and processes (predicates) "exist" relative to ontological spaces defined by their coordinates on the space (s), time (t) and modality (m) axes $^{2}$. (Chilton, 2004, p.57-58)

Pour P. Chilton, les discours politiques sont des terrains particulièrement favorables à ce type d'analyse, dans la mesure où les positionnements des espaces déictiques (internes, pour soi ; externes, pour les autres) forment les bases pragmatiques et sémantiques des représentations. Nous proposons, sur la base de ces éléments, de délimiter d'abord les espaces déictiques présents dans le sous-corpus de l'exécutif français :

- «Nous», «nos» et «nôtres» représentent l'espace déictique interne de l'État républicain français, et se retrouvent principalement liés aux lexèmes «pays» (17 cooccurrences), «contre» (15 cooccurrences), «France» (10 cooccurrences), «terrorisme» (10 cooccurrences), «frontières» (9 cooccurrences), «concitoyens » (8 cooccurrences), «valeurs » (8 cooccurrences) et «territoire» (7 cooccurrences);

- «Je » et «j” » représentent l'espace déictique interne du président François Hollande, et se retrouvent principalement liés aux lexèmes «vous» (12 cooccurrences) et «président» (4 cooccurrences);

- «Terroristes», "terroriste» et «Daech» représentent l'espace déictique externe principal, contre lequel se dirigent les discours du locuteur, et se retrouvent liés aux lexèmes «armée» (9 cooccurrences), «contre» (7 cooccurrences), «nous» (6 cooccurrences), «actes» (5 cooccurrences), «menace» (4 cooccurrences), «Syrie» (4 cooccurrences) et « groupe» (4 cooccurrences).

2. «Les entités, ainsi que les relations entre elles, peuvent être représentées comme physiquement nécessaires, socialement imposées ou moralement impératives. Il y a également un élément spatial fort dans cette dimension. [...] Nous estimons que, lors du traitement du discours, les individus "positionnent" d'autres entités dans leur "monde" en "positionnant" ces entités en relation avec eux-mêmes sur trois axes (au moins), à savoir l'espace, le temps et la modalité. Le centre déictique (le Moi, c'est-à-dire je ou nous) constitue “l’origine” de ces trois dimensions. D’autres entités (les arguments de prédicats) et processus (prédicats) "existent" relativement aux espaces ontologiques définis par leurs coordonnées sur les axes de l'espace (e), le temps $(t)$ et la modalité $(m)$.» 
Les espaces potentiels délimités par «ils » ou «vous» ne sont pas assez significatifs en termes de cooccurrences sémantiques pour être ici représentés, mais cela n'empêche en rien l'analyse des espaces ainsi délimités pour l'exécutiffrançais : en effet, la personne seule du «je », à savoir le chef de l'État, se fond au sein d'un «nous » derrière lequel il disparaît, pour parler au nom de l'État et du peuple français, saisissant par là même la parole officielle attachée à son rôle de président pour l'ancrer dans un imaginaire collectif qui se substitue à la pluralité des analyses possibles du peuple. Ce faisant, de manière axiologique, il positionne un pays entier contre un groupe terroriste, qui est immédiatement identifié à la possibilité menaçante d'attentats. Nous pouvons représenter la tension entre ces espaces déictiques à l'aide du schéma suivant, en suivant les axes temporel (AT), spatial (AS), modal (AM) et axiologique (AA).

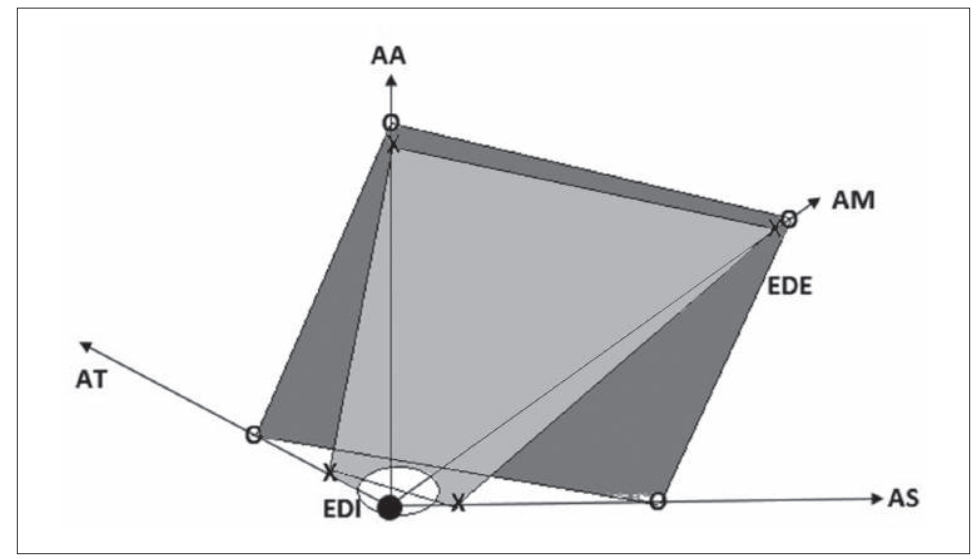

Schéma 7 : modalisation des espaces déictiques du sous-corpus France

Cette modélisation nous indique plusieurs éléments : nous avons souhaité représenter l'espace déictique interne comme incluant par un cercle blanc le «je » présidentiel, inclus dans un «nous » qui dépasse sa fonction. Cet espace déictique interne se déploie sur des axes temporel et spatial proches, puisqu'il s'agit de se situer dans l'ici et maintenant pour pouvoir se projeter contre un ennemi qui est d'abord éloigné du point de vue axiologique. Du point de vue modal, l'espace déictique interne se retrouve en situation d'action forte, avec des verbes modaux particulièrement importants, que nous pouvons retrouver dans les exemples suivants :

1. Nous sommes dans une guerre contre le terrorisme

2. Nous poursuivrons ces frappes au cours des semaines à venir

3. Le terrorisme, nous le combattons partout là où des États sont menacés

4. Nous devons être impitoyables

5. Nous devons donc nous défendre dans l'urgence et dans la durée 
Ces exemples de modalisation illustrent une autre réalité, que nous avons souhaité représenter dans le schéma ci-dessus : du point de vue axiologique, si la mise en discours de l'incompatibilité des valeurs des terroristes et d'un pays comme la France semble facile à déceler, la rhétorique guerrière pourtant attribuée aux auteurs des attentats se retrouve d'ores et déjà dans la politique antiterroriste internationale menée par l'État français, et se poursuit très largement dans les occurrences mettant en discours l'espace déictique interne. Si l'argumentation de la violence terroriste de l'espace déictique externe est bien présente, l'espace déictique interne semble vouloir rejoindre l'espace déictique externe, représenté de manière plus éloignée mais pourtant plus étendue sur les quatre axes. Du point de vue discursif, tout se passe comme si le «nous» imaginé et représenté par le président François Hollande devait aller chercher et combattre les terroristes avec les mêmes armes et la même violence qu'eux.

Les espaces déictiques représentés dans les discours produits par l'exécutif belge, à savoir le Premier ministre Charles Michel et le roi Philippe de Belgique, présentent des caractéristiques fort différentes, induisant par là même des représentations fort singulières du monde post-attentats :

- «Nous», «nos» et "nôtres» représentent l'espace déictique interne de l'État belge, et se retrouvent liés aux lexèmes "pays» (8 cooccurrences), "population» (3 cooccurrences), "sécurité» (3 cooccurrences), «capitale» (2 cooccurrences), "concitoyens» (2 cooccurrences), "aéroport» (2 cooccurrences) et «valeurs» (2 cooccurrences);

- «Leurs» représente l'espace déictique externe des victimes des attentats, et se retrouve mis en discours avec les lexèmes «proches» (5 cooccurrences), «compagnes/compagnons» (4 cooccurrences), «familles» (4 cooccurrences), «victimes» (2 cooccurrences), «corps» (2 cooccurrences), «cœurs» (2 cooccurrences).

Le pronom «je», qui représente l'espace déictique interne des deux représentants de l'exécutif, se trouve trop faiblement représenté dans le corpus pour pouvoir être significatif du point de vue de l'analyse. Quant aux auteurs des attaques, ils se retrouvent relativement sous-représentés au sein des discours du Premier ministre ou du roi de Belgique. L'espace déictique externe, quant à lui, n'est pas représenté par les pronoms personnels traditionnels comme «ils» ou «elles», mais par un possessif qui rend d'autant plus palpable leur place au sein de la société ou de la cellule familiale, et donc d'autant plus tragiques les pertes humaines. De ce point de vue, la différence avec les discours de l'exécutif français est profonde : là où le président François Hollande a pour cible discursive les terroristes et la lutte contre ce qu'ils représentent, aux niveaux national comme international, l'exécutif belge a d'abord pour cible sa propre population, meurtrie par les attentats, comme le montre la modélisation suivante. 


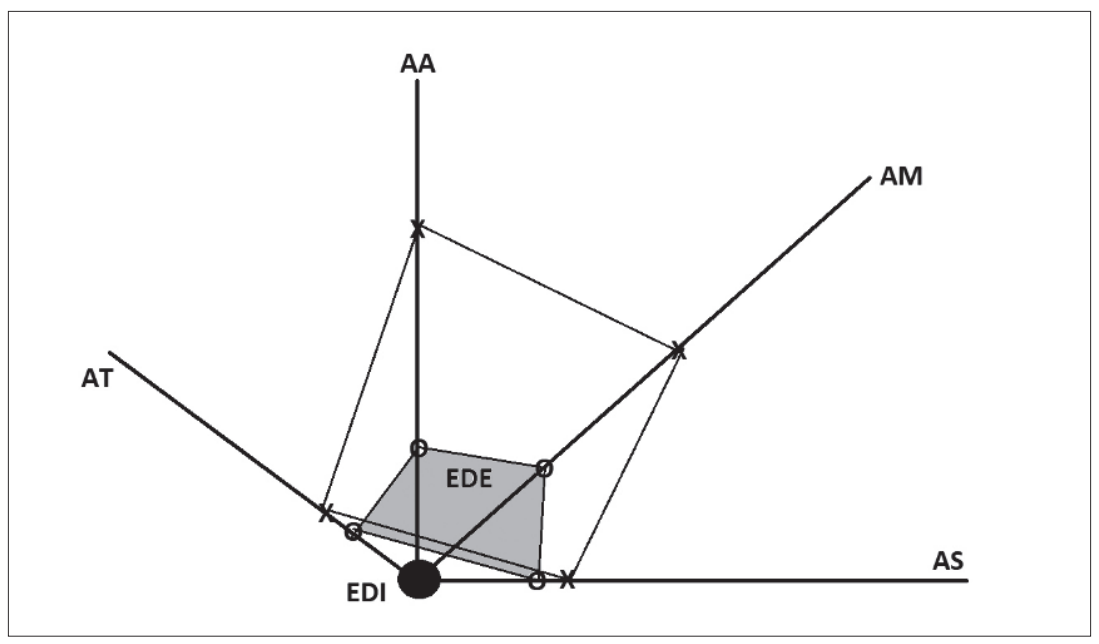

Schéma 8 : modalisation des espaces déictiques du sous-corpus Belgique

La modélisation qui résulte de cette analyse des espaces déictiques présente, de fait, une spatialisation nettement différente. En s'adressant à sa propre population, l'exécutif belge s'adresse au cœur de son pays et tente de lui parler en le rassurant, afin d'essayer d'adoucir le traumatisme des attentats. Ainsi, l'espace déictique externe ciblé par les discours de l'exécutif se retrouve au sein même de l'espace déictique interne (en zone grisée sur la présente modélisation), avec des positionnements axiologiques et modalisateurs les plus proches possibles de la vie sociale quotidienne du pays, touchant ainsi chaque citoyen dans son humanité. L'espace déictique interne se retrouve modélisé de manière proche sur les axes spatial et temporel, mais s'éloigne des points de vue modal et axiologique en assumant un rôle de sécurité et de gardien des valeurs, sans prétendre cependant pouvoir embrasser la douleur des victimes, comme nous pouvons le voir à travers les exemples suivants :

6. Notre pays et nos concitoyens ont été frappés par des attentats

7. Nous voulons à nouveau exprimer notre compassion vis-à-vis des victimes

8. Face à cette barbarie notre population a instantanément choisi de faire bloc

9. La première urgence pour nous est évidemment le traitement des victimes 10. Nous devons faire face à cette épreuve en étant unis, en étant solidaires, en étant rassemblés

Ces prélèvements syntaxiques tranchent, s’il était besoin de le souligner, avec ceux que nous avons choisi d'exposer pour représenter l'espace déictique interne de l'exécutif français et achèvent de mettre en exergue les importantes différences de réaction entre deux États face à des événements aussi tragiques que sont des attentats terroristes imprévus et aveugles. 


\section{Discussion}

Face aux attentats terroristes, il n'existe pas une seule réponse de l'exécutif politique dans les démocraties occidentales. Nous l'avons mis partiellement en lumière grâce à la présente étude, en comparant les réactions discursives de deux exécutifs, issus de deux partis politiques différents, et enracinés dans deux États dont les histoires sociales, politiques et internationales sont singulières à plus d'un titre. La topographie des nœuds sémantiques, la modélisation des espaces déictiques ou les dendrogrammes des classes sémantiques l'ont montré : là où l'exécutif français tente de dépasser les attentats pour justifier auprès de sa population la lutte contre le terrorisme, quitte à justifier ses interventions armées a posteriori, notamment en Irak, l'exécutif belge prend d'abord le temps de se tournervers sa population, dans une forme d'empathie discursive en lien avec la souffrance légitime des victimes. Dans le premier cas, la réponse au terrorisme s'incarne dans une lutte armée, qui cadre avec le rôle de la France dans le monde comme puissance militaire; dans le second cas, la réponse au terrorisme s'exprime à travers la préservation d'un vivre-ensemble fortement mis à mal par une tragédie imprévisible - peut-être est-ce la seule réponse possible pour un petit État européen qui n'a pas les moyens militaires et diplomatiques de la France. Toutefois, comme il s'agit ici de discours politiques, il nous semble important de revenir sur la contextualisation propice à la manipulation, en tant que processus cognitif et communicationnel de mise en place des conditions de sélection de l'information :

Manipulative communication is a twofold process by which a constraint that limits context selection is combined with a target utterance $U$ in order to force the interpretation of the latter within a limited set of contextual assumptions and to effectively ensure that the interpretation is reached before a known, alternative (contradictory) subset of assumptions is accessed. [...] Manipulation works on making a context $C$, in which $U$ is strengthened and gives rise to contextual implications, much more accessible, thereby effectively blocking access to C3. (Maillat, 2013, p.195)

Pour les deux sous-corpus que nous avons choisi d'étudier, cela se traduit par la construction des contextes suivants, qui visent à renforcer les occurrences cibles telles que décrites par Didier Maillat:

- Dans le cas de l'exécutif français, il s'agit de sensibiliser la population à une lutte externe contre le terrorisme et à une protection de la population fran-

3. «La communication manipulatoire est un processus double dans lequel une contrainte limitant la sélection du contexte est combinée avec une occurrence cible $O$, afin de forcer l'interprétation de cette dernière au sein d'un ensemble limité d'hypothèses contextuelles, mais également d'assurer de façon effective que l'interprétation soit atteinte avant qu'un sous-ensemble d'hypothèses alternatif (contradictoire) et connu soit atteint. [...] La manipulation rend bien plus accessible un contexte $C$, dans lequel $O$ est renforcé et provoque l'émergence d'implications contextuelles, bloquant ainsi l'accès effectif à $C$. » 
çaise par les frontières, gommant ainsi le fait que la plupart des terroristes qui ont frappé cette dernière ont la nationalité française, et que le terrorisme islamique a précisément été alimenté par la politique étrangère de la France.

- Dans le cas de l'exécutif belge, mettre en lumière le vivre-ensemble et la compassion envers la victime revient à tenter d'éviter de provoquer l'éclatement d'une démocratie déjà fragilisée par ses dissensions linguistiques internes, et aussi de taire les difficultés liées à la radicalisation et à l'organisation de cellules terroristes dans des quartiers de Bruxelles comme Molenbeek.

L'exécutif politique, en tentant de masquer ou de minimiser les failles de son propre traitement de la menace d'attentat sur son sol, menace à laquelle il participe inévitablement par les choix qu'il fait, utilise donc des stratégies d'argumentation discursives qui sont en accord avec des orientations politiques nationales et souvent historiques (militaires et internationales, par exemple, dans le cas de la France); cette stratégie de légitimation des choix de l'État vise non seulement à éviter de le fragiliser en période de crise, mais également à conjurer l'émergence d'interprétations discordantes par la réactivation de valeurs nationales et l'instrumentalisation émotionnelle. Orientée vers la réaction sécuritaire dans un cas ou la compassion dans l'autre, cette stratégie de légitimation semble être l'une des seules réponses apportées aux citoyens victimes d'attentats.

\section{Références}

BAKER Paul, 2006, Using Corpora in Discourse Analysis, Londres, Continuum.

Bendinelu Marion, 2011, «Anglais de spécialité et logométrie. L'exemple des débats présidentiels américains », $A S p$, n60, p. 103-123.

Boudon Raymond, 1995, Le juste et le vrai, Paris, Fayard.

CAP Piotr, 2013, "Proximization theory and critical discourse studies: a promising connection? », International Review of Pragmatics, no $5 / 2$, p. 293-317.

Chilton Paul, 2004, Analysing Political Discourse. Theory and Practice, Londres, Routledge.

GalATANu Olga, 2009, «L'analyse du discours dans la perspective de la sémantique des possibles argumentatifs : les mécanismes sémantico-discursifs de construction du sens et de reconstruction de la signification lexicale», dans L'analyse linguistique des corpus discursifs. Des théories aux pratiques, des pratiques aux théories, N. Garric et J. Longhi éd., Clermont-Ferrand, Presses universitaires Blaise Pascal, p. 49-38.

GARRIC Nathalie, LONGHI Julien, 2013, «Atteindre l'interdiscours par la circulation des discours et du sens», Langage et société, n0144/2, p. 65-83.

KopytowsKa Monika, 2015, «Mediating identity, ideology and values in the public sphere: towards a new model of (constructed) social reality », Lodz Papers in Pragmatics, $\mathrm{n}^{0} 11 / 2$, p.133-156.

KRIEg-Planque Alice, Oger Claire, 2010, «Discours institutionnels. Perspectives pour les sciences de la communication », Mots. Les langages du politique, $\mathrm{n}^{\circ}$ 94, p. 91-97. LAZORTHES Frédéric, 2006, « Le libéral et la constitution de la Ve République : Aron et le 
complexe français du pouvoir exécutif », Droits. Revue française de théorie, de philosophie et de culture juridiques, nº 44/2, p. 59-70.

MAILlAT Didier, 2013, «Constraining context selection: on the pragmatic inevitability of manipulation », Journal of Pragmatics, n5 59/2, p. 190-199.

O'BRIEN Peter, 2016, «Counter-terrorism in Europe: the elusive search for order », European Security, $n^{\circ}$ 25/3, p. 366-384.

SARFATI George-Elia, 2014, "L'emprise du sens : note sur les conditions théoriques et les enjeux de l'analyse du discours institutionnel», dans Les discours institutionnels en confrontation. Contribution à l'analyse des discours institutionnels et politiques, J. Longhi et G.-E. Sarfati éd., Paris, L'Harmattan, p.13-46.

UytTendaele Marc, 2011, "Chronique d'une législature maudite. Réflexions sur l'instabilité politique de la Belgique», Pouvoirs. Revue française d'études constitutionnelles et politiques, $n^{\circ} 136 / 1$, p. 51-69. 